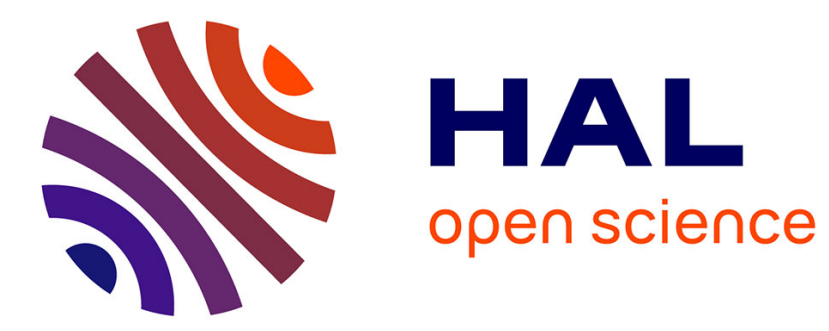

\title{
Femtosecond fiber chirped- and divided-pulse amplification system
}

Yoann Zaouter, Florent Guichard, Louis Daniault, Marc Hanna, Franck

Morin, Clemens Hönninger, Eric Mottay, Frédéric Druon, Patrick Georges

\section{- To cite this version:}

Yoann Zaouter, Florent Guichard, Louis Daniault, Marc Hanna, Franck Morin, et al.. Femtosecond fiber chirped- and divided-pulse amplification system. Optics Letters, 2013, 38 (2), pp.106-108. hal01303618

\section{HAL Id: hal-01303618 \\ https://hal-iogs.archives-ouvertes.fr/hal-01303618}

Submitted on 19 Apr 2016

HAL is a multi-disciplinary open access archive for the deposit and dissemination of scientific research documents, whether they are published or not. The documents may come from teaching and research institutions in France or abroad, or from public or private research centers.
L'archive ouverte pluridisciplinaire HAL, est destinée au dépôt et à la diffusion de documents scientifiques de niveau recherche, publiés ou non, émanant des établissements d'enseignement et de recherche français ou étrangers, des laboratoires publics ou privés. 


\title{
Femtosecond fiber chirped- and divided-pulse amplification system
}

\author{
Yoann Zaouter, ${ }^{1, *}$ Florent Guichard, ${ }^{1,2}$ Louis Daniault, ${ }^{2}$ Marc Hanna, ${ }^{2}$ Franck Morin, ${ }^{1}$ \\ Clemens Hönninger, ${ }^{1}$ Eric Mottay, ${ }^{1}$ Frédéric Druon, ${ }^{2}$ and Patrick Georges ${ }^{2}$ \\ ${ }^{1}$ Amplitude Systemes, 11 avenue de Canteranne, Cité de la Photonique, Pessac 33600, France \\ ${ }^{2}$ Laboratoire Charles Fabry, Institut d'Optique, CNRS, Université Paris-Sud, 2 Ave. Augustin Fresnel, Palaiseau Cedex 91127, France \\ *Corresponding author: yzaouter@amplitude-systemes.com
}

\begin{abstract}
We implement both chirped pulse amplification and divided pulse amplification in the same femtosecond fiber amplifier setup. This scheme allows an equivalent stretched pulse duration of $2.4 \mathrm{~ns}$ in a compact tabletop system. The generation of $77 \mathrm{~W}$ of compressed average power at $4.8 \mathrm{MHz}$, together with $320 \mathrm{fs}$ and $430 \mu \mathrm{J}$ pulses at a repetition rate of $96 \mathrm{kHz}$, is demonstrated using a distributed mode-filtering, rod-type, ytterbium-doped fiber. Limitations in the temporal recombining efficiency due to gain saturation inside the fiber amplifier are identified.
\end{abstract}

A very efficient way of scaling the energy of amplified femtosecond pulses was provided by the chirped-pulse amplification (CPA) method [1]. This technique consists in temporally spreading the ultrashort pulse prior to amplification using a highly dispersive optical setup, thereby reducing the peak power by up to four orders of magnitude. This process can be reversed after the amplification to reveal the true amplified peak power. Although CPA is used in virtually every amplified femtosecond system, its implementation is increasingly difficult and costly when the desired stretched pulse duration is greater than $1 \mathrm{~ns}$, due to the increasing size of the gratings and overall optical setup. Such long stretched pulse durations are therefore not used for compact tabletop or industrial systems.

The divided-pulse amplification (DPA) idea was proposed more recently [2] and is similar to CPA in the sense that it also consists in redistributing the pulse energy over a time interval larger than the initial pulse width to reduce the peak power. In this scheme, a train of several orthogonally polarized delayed pulse replicas is generated and amplified before final recombination. The technique has been successfully implemented to amplify picosecond pulses [3,4], for which it is difficult to use the CPA method because of the small optical bandwidth available. It has also been used to amplify femtosecond pulses in the parabolic regime [5], which necessitates unchirped pulses at the input of the amplifier. In both cases the delay between two successive replicas was of the order of a few picoseconds and was therefore advantageously realized using centimeter-long, highly birefringent vanadate crystals.

In this Letter, we propose and demonstrate for the first time a femtosecond fiber amplifier that uses both concepts simultaneously to scale the output energy of a tabletop femtosecond fiber system. Because the CPA stretched-pulse duration is of the order of hundreds of picoseconds, the DPA delays are implemented using freespace interferometers with reasonable arm lengths of up to $1.32 \mathrm{~m}$. Because the energy of the amplified pulses is not negligible compared to the saturation energy of the fiber amplifier, a decrease of the temporal combining efficiency appears, related to the different levels of saturation of the gain medium seen by the replicas in the train, leading to different phase accumulations due to self-phase modulation (SPM) and Kramers-Krönig $(\mathrm{K}-\mathrm{K})$ effects. Despite this limitation, our experiment allows the generation of $320 \mathrm{fs}, 430 \mu \mathrm{J}$ pulses at a repetition rate of $96 \mathrm{kHz}$, corresponding to $42 \mathrm{~W}$ average power, using a distributed mode filtering (DMF) rod-type large-mode-area fiber [ 6 ] with a combining efficiency of $82 \%$.

The $1.2 \mathrm{~m} \times 0.4 \mathrm{~m}$ footprint experimental arrangement is depicted in Fig. 1. The DPA setup is included inside a moderately nonlinear fiber CPA in which the impact of nonlinearities can be partially compensated by the dispersion mismatch of the stretcher and compressor units [7]. The front end is composed of a passively modelocked ultrafast oscillator, a pulse picker, a stretcher, and a low-power fiber preamplifier. The oscillator generates pulses at the central wavelength of $1030 \mathrm{~nm}$ and pulse duration of $200 \mathrm{fs}$. The repetition rate of the oscillator of $25 \mathrm{MHz}$ is divided by an acousto-optic modulator pulse picker. In this experiment, two repetition rates of $4.8 \mathrm{MHz}$ and $96 \mathrm{kHz}$ are selected to investigate the linear and nonlinear regimes, respectively. The stretching ratio is designed to preserve a pulse duration above $500 \mathrm{ps}$ after the power amplifier when operated at maximum gain. The low-power fiber preamplifier is used to boost the average power to a few tens of $\mathrm{mW}$ to ensure efficient operation of the power amplifier. An optical isolator is placed after the front end to prevent any optical feedback

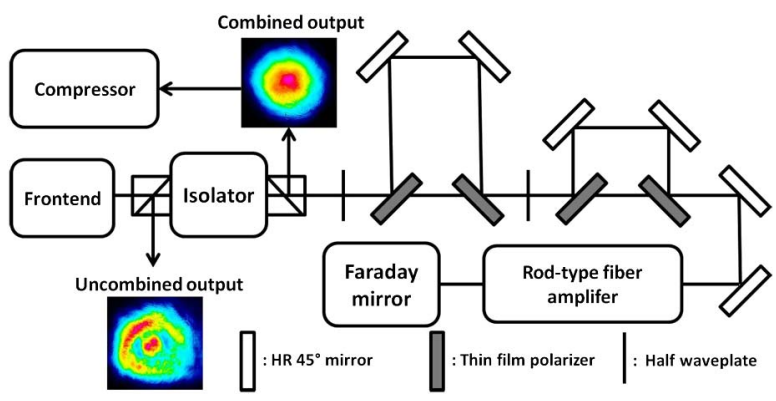

Fig. 1. (Color online) Experimental setup. 
to the front end coming from the power amplifier. Temporal pulse divisions are implemented using two freespace Mach-Zehnder interferometers in which the splitting and recombining elements are thin-film polarizers. Depending on the orientation of the input linear polarization, the power fraction sent in each arm can be adjusted. In particular, one arm can be completely bypassed, reducing the number of replicas generated. The arm-length differences in the interferometers are 1.32 and $0.66 \mathrm{~m}$, respectively. On the way to the amplifier, this arrangement therefore generates a train of up to four orthogonally polarized chirped-pulse replicas separated by a delay of $2.2 \mathrm{~ns}$. Therefore, the equivalent pulse stretching ratio of this system can be modulated up to $2.4 \mathrm{~ns}$ at maximum replica counts. The fiber power amplifier is made of a $1 \mathrm{~m}$ long, $85 \mu \mathrm{m}$ core, $250 \mu \mathrm{m}$ clad, DMF rod-type fiber pumped by a $180 \mathrm{~W}$ fiber-coupled high-power diode at $976 \mathrm{~nm}$. In order to temporally recombine the pulse replicas, they are amplified in a double-pass geometry, and a Faraday rotator is used after the first pass to flip the polarization states by $90^{\circ}$. After the second pass, the amplified pulses propagate backward through the Mach-Zehnder interferometers, and the delays accumulated to create the replicas are canceled to generate a single stretched pulse. The polarizer associated with the isolator output extracts the amplified beam, while the polarizer at the input extracts the residual beam with orthogonal polarization. The amplified beam is finally compressed using a high-efficiency grating compressor possessing $80 \%$ throughput.

The setup is first operated at a high repetition rate of 4.8 MHz. The combined, uncombined, and compressed average powers at the output are plotted along with the temporal combining efficiency (defined as the ratio between the combined average power and the total output power) as a function of pump power in Fig. $\underline{2}$ (top row). With two replicas activated [see Fig. 2(a)], the combining efficiency starts at $98 \%$ and remains above $97 \%$ for
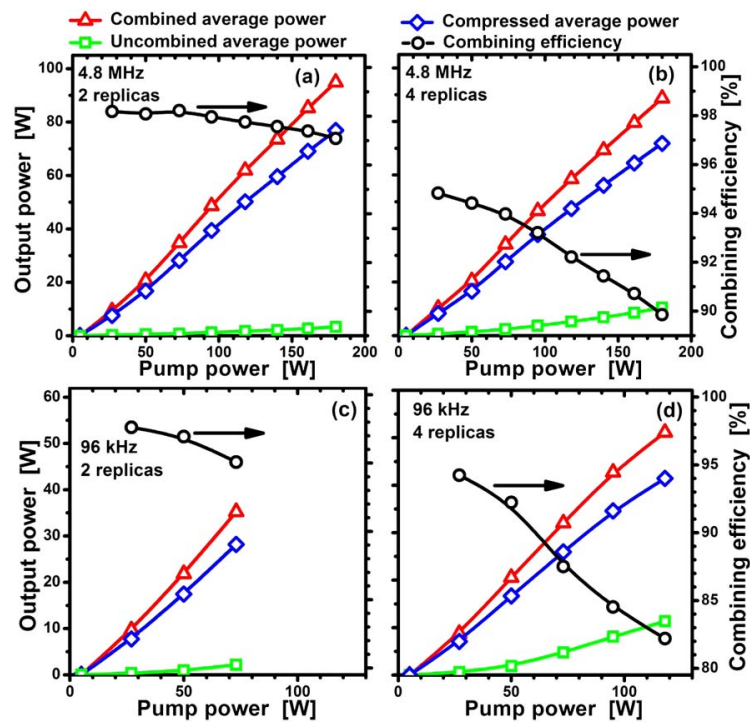

Fig. 2. (Color online) Combined, uncombined, and compressed average power and temporal combining efficiency as a function of pump power for $96 \mathrm{kHz}$ (bottom) and $4.8 \mathrm{MHz}$ (top) repetition rates, and 2 (left) and 4 (right) replicas. all pump powers. When four replicas [see Fig. 2(b)] are generated, the combining efficiency starts at a lower value of $95 \%$ and decreases to just below $90 \%$ at the highest pump power. The maximum compressed average power is $77 \mathrm{~W}$ with two replicas, and $72 \mathrm{~W}$ with four replicas. The physical origin of the nonperfect combining at low power is identified by looking at the spatial profiles of the combined and rejected beams, displayed in Fig. 1 . A circular fringe pattern is caused by the coherent superposition of beams that have propagated over different distances (different arm lengths on the way back), imparting slightly different radii of curvature to the wavefronts. This effect is more pronounced when the number of replicas increases because the differential propagation distance between the replicas increases accordingly. If this limitation becomes too important, imaging techniques could be implemented to solve it. The decrease of combining efficiency as the pump power increases is probably related to slight mechanical misalignments induced by thermal effects. These thermal effects also impact the long-term stability of the system, which remains better than $1 \%$ rms over $1 \mathrm{~h}$. This stability could easily be improved with properly temperature regulated optomechanics. Also, for all investigated parameters, no pulse-to-pulse stability degradation has been noticed.

The repetition rate is then set to $96 \mathrm{kHz}$ to operate the setup in the high-pulse-energy regime. To confirm the reduction of nonlinear effects induced by the DPA setup, we compare the pulse autocorrelations at a fixed output energy of $175 \mu \mathrm{J}$ after compression for one, two, and four replicas (Fig. 3). In each case, the compressor is adjusted to optimize the compressed pulse duration. Without any DPA replicas, the compressed pulse exhibits low shoulders and a pedestal, typical of uncompensated higher-order nonlinear spectral phase due to SPM in the fiber amplifier. When two replicas are used, there is a clear improvement in peak power and the shoulders and pedestal disappear, indicating a lower accumulated nonlinear phase. Further increase in the number of replicas results in a marginal improvement of the pulse temporal quality. This confirms that DPA allows energy scaling of the setup.

The power characteristics of the setup at $96 \mathrm{kHz}$ repetition rate are shown in Fig. $\underline{2}$ (bottom row). As expected, the combining efficiency at low power is unchanged compared to the high-repetition-rate case. However, when the energy is scaled, we observe an additional

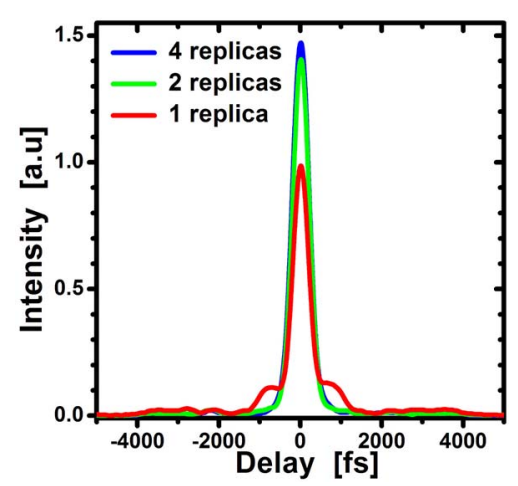

Fig. 3. (Color online) Autocorrelation traces measured at $175 \mu \mathrm{J}$ total output energy for one, two, and four pulse replicas. 

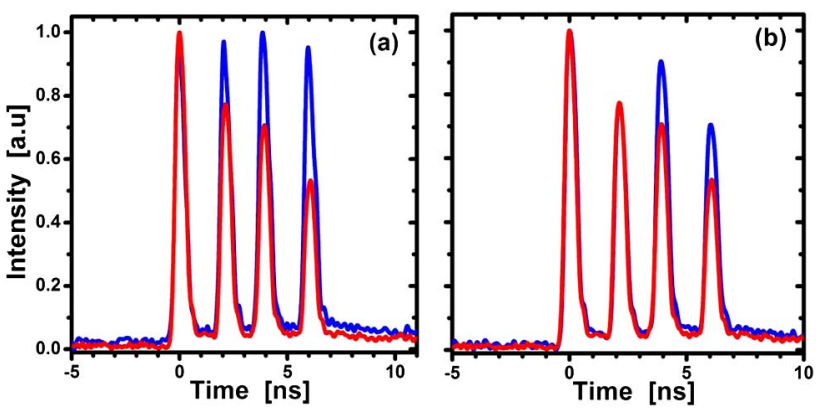

Fig. 4. (Color online) Oscilloscope temporal traces of the train of four replicas at the input (blue curve) and output (red curve) of the amplifier. Left: equal energy seeding. Right: optimal energy seeding at the highest pulse energy of $430 \mu \mathrm{J}$.

decrease in efficiency clearly related to the high output pulse energy and more important when four replicas are generated. To investigate the origin of this efficiency loss, a $4 \mathrm{GHz}$ sampling oscilloscope is used to measure the temporal profile on the nanosecond time scale at the input and output of the amplifier, after temporal splitting and before temporal recombination. When the waveplates associated with each Mach-Zehnder are set to equally distribute the power over the replicas at the input (Fig. $\underline{4}$, left), we clearly observe saturation of the gain for high-energy operation, leading to temporally decreasing amplified pulse energy. This decreasing energy distribution can in turn be translated into a differential phase among the replicas by two mechanisms: SPM and K-K coupling between gain and refractive index along the fiber. Overall, both the energy and phase of the replicas are modified by the saturation. To evaluate the phase induced by SPM and K-K, we only activate two replicas in the system and measure the energies of each replica at the output, for equal energy seeding, that corresponds to a combining efficiency of $80 \%$. At this point the average output energy per replica is $100 \mu \mathrm{J}$, and the energy difference between both replicas is $25 \%$. Taking into account the spatial (3\%) and thermally induced mechanical drift (2\%) contributions to the efficiency loss, the overall saturation-induced phase is evaluated to be $0.8 \mathrm{rad}$. Although it should be investigated in more detail, rough estimations indicate that both SPM and K-K effects contribute significantly to the differential phase.

A simple way to counterbalance the gain saturation is to adjust the waveplates associated with each DPA division to maximize the efficiency. The combining efficiency plotted in Fig. 2 corresponds to this optimization. For two replicas, the waveplate can be adjusted to mostly compensate for saturation, resulting in a combining efficiency above $95 \%$ at all energy levels. For four replicas, however, three relative energies must be adjusted with only two degrees of freedom, and the compensation is less efficient, leading to $82 \%$ efficiency at the highest overall energy. We finally investigate the temporal and spectral properties of the final pulse at maximum energy with four replicas [see Fig. 2(d)]. At $96 \mathrm{kHz}$, the maximum average power measured after the compressor is $42 \mathrm{~W}$ ( $52 \mathrm{~W}$ before), that is, $430 \mu \mathrm{J}$ of energy per pulse.

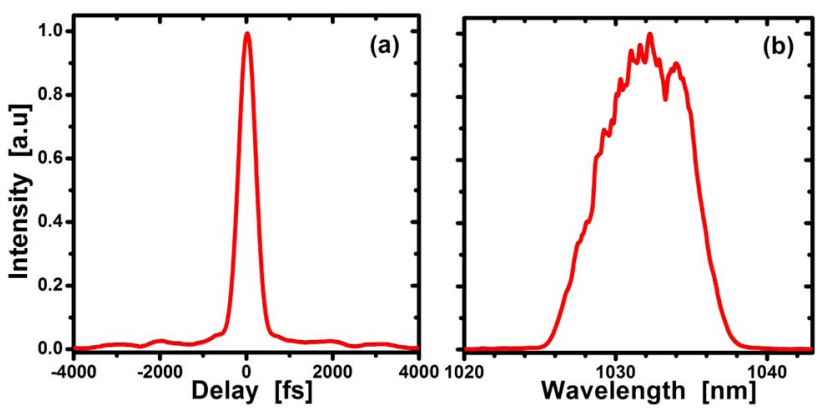

Fig. 5. (Color online) Autocorrelation trace (left) and spectrum (right) measured at $430 \mu \mathrm{J}$.

Figure 5 shows the autocorrelation trace and spectrum of the pulse. The autocorrelation FWHM is $450 \mathrm{fs}$, corresponding to a $320 \mathrm{fs}$ pulse if a Gaussian shape is assumed. The spectral width is $7 \mathrm{~nm}$, leading to a time-bandwidth product of 0.57 , and the estimated peak power is in excess of $1 \mathrm{GW}$. We verify on the oscilloscope trace taken after temporal combining that the state of polarization of the replicas is well preserved in the amplifier, so that the parasitic pulses observed at the output in the time domain contribute less than $1 \%$ to the total energy. Finally, we analyze the beam quality after compression. $M^{2}$ measurements are systematically carried out and show no degradation of the beam quality with increasing combined average power with typical values of $M_{x}^{2}=1.25$ and $M_{y}^{2}=1.20$.

In conclusion, we demonstrate that CPA and DPA can be successfully implemented together to reach equivalent stretched pulse widths, or peak-power reduction factors, that are not accessible in tabletop fiber CPA femtosecond systems. The use of DMF, Yb-doped, rod-type fibers permits generating $77 \mathrm{~W}$ of compressed average power at $4.8 \mathrm{MHz}$, and ultrashort 320 fs pulses with $430 \mu \mathrm{J}$ of energy per pulse at a repetition rate of $96 \mathrm{kHz}$. Similarly to $[4,5]$, the CPA-DPA architecture could be implemented together with a Sagnac interferometer to further reduce the energy per replica and sensitivity to gain saturation, and to allow the use of a polarizing gain medium. We strongly believe this technique will allow further energy scaling of tabletop femtosecond systems.

\section{References}

1. D. Strickland and G. Mourou, Opt. Commun. 56, 219 (1985).

2. S. Zhou, F. W. Wise, and D. G. Ouzounov, Opt. Lett. 32, 871 (2007).

3. L. J. Kong, L. M. Zhao, S. Lefrancois, D. G. Ouzounov, C. X. Yang, and F. W. Wise, Opt. Lett. 37, 253 (2012).

4. S. Roither, A. J. Verhoef, O. D. Mücke, G. A. Reider, A. Pugzlys, and A. Baltuska, Opt. Express 20, 25121 (2012).

5. L. Daniault, M. Hanna, D. N. Papadopoulos, Y. Zaouter, E. Mottay, F. Druon, and P. Georges, Opt. Express 20, 21627 (2012).

6. M. Laurila, M. M. Jørgensen, K. R. Hansen, T. T. Alkeskjold, J. Broeng, and J. Lægsgaard, Opt. Express 20, 5742 (2012).

7. Y. Zaouter, J. Boullet, E. Mottay, and E. Cormier, Opt. Lett. 33, 1527 (2008). 